\title{
Full-wave electromagnetic and thermal modeling for the prediction of heat-dissipation-induced RF-MEMS switch failure*
}

\author{
Zhongde Wang ${ }^{1}$, Brian D Jensen ${ }^{2}$, Linda L W Chow ${ }^{2}$, \\ John L Volakis ${ }^{3}$, Kazuhiro Saitou ${ }^{2}$ and Katsuo Kurabayashi ${ }^{2}$ \\ ${ }^{1}$ Electrical Engineering and Computer Science Department, University of Michigan, \\ Ann Arbor, MI 48109, USA \\ ${ }^{2}$ Mechanical Engineering Department, University of Michigan, Ann Arbor, MI 48109, USA \\ ${ }^{3}$ Electrical and Computer Engineering Department, The Ohio State University, Columbus, \\ OH 43210, USA
}

E-mail: zhongdew@eecs.umich.edu, volakis.1@osu.edu and katsuo@umich.edu

Received 22 June 2005, in final form 1 November 2005

Published 16 December 2005

Online at stacks.iop.org/JMM/16/157

\begin{abstract}
We propose an extended finite element-boundary integral method (EFE-BI) to model the electromagnetic (EM) behavior of RF-MEMS switches over a wide frequency range from UHF to terahertz. Our new method integrates EM with finite element heat transfer analysis to extract heat dissipation on the micrometer-scale switch beam due to the non-uniform radio frequency (RF) current distribution. The developed EFE-BI technique is an extension of the standard finite element-boundary integral (FE-BI) method to allow for accurate characterization of RF-MEMS structures whose entire size is a small fraction of a wavelength $(\lambda / 250$ or less) and may contain dimensions in the order of $\lambda / 50000$ or less. Our model predictions exhibit good agreement with experimental results obtained independent of the current study.
\end{abstract}

\section{Introduction}

RF-MEMS switches have demonstrated low on-state insertion loss, high off-state isolation and very linear behavior [1-6]. Despite these excellent characteristics, they generally suffer from low power-handling capability, with most switches operating well below $1 \mathrm{~W}$ [4]. This limitation is due to the complex interactions among the electromagnetic losses, heat transfer and mechanical deformations. To better understand the associated switch failure mechanisms, a multiphysics model is required to capture the coupling among electromagnetic, thermal and mechanical domains [7]. As a step towards this end, this paper presents the development of an electromagnetic model that allows for accurate modeling within a small volume surrounding a MEMS switch while

* This work was supported in part by the National Science Foundation under grant no ECS-01152222. still addressing a wide range of frequencies from 0.5 and $3000 \mathrm{GHz}$. The integration of this electromagnetic model with a heat transfer analysis will allow for the prediction of the failure mechanisms associated with MEMS switches at elevated temperature.

We select an inline dc-contact MEMS shunt switch [4] as illustrated in figure 1 to most effectively demonstrate the capability of the developed analysis. The device is attached to an underlying substrate and consists of a fixed-fixed micromachined beam 100-600 $\mu \mathrm{m}$ long, suspended above the substrate by a gap of $1-3 \mu \mathrm{m}$, typically intended for frequencies ranging from $1 \mathrm{GHz}$ to several hundred $\mathrm{GHz}$. The up-state operation of this particular switch design is expected to suffer most from heat-induced failure mechanisms among existing RF-MEMS switch designs. An assessment of the power-handling capability and reliability of the switch will therefore benefit from the model developed in this work. 


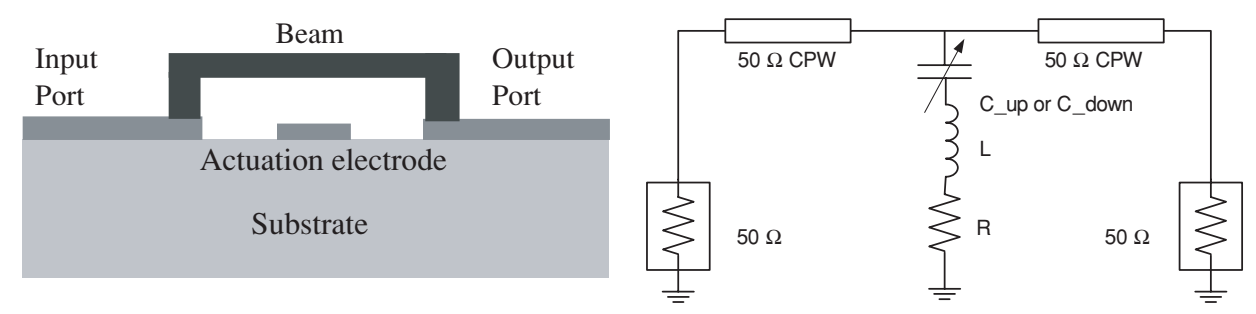

Figure 1. An RF-MEMS inline shunt switch and its lumped circuit model.

A challenge in our modeling relates to the extremely small dimensions of the MEMS switch. More specifically, at $2 \mathrm{GHz}$, MEMS dimensions correspond to an electrical length of $\lambda / 1500$ to $\lambda / 250$ and a gap of $\lambda / 150000$ to $\lambda / 50000$. The total size of the MEMS device is thus many times smaller than a typical element size used in finite element (FEM) or finite difference-time domain (FDTD) codes (approximately $\lambda / 20$ to $\lambda / 10)[8,9]$. Therefore, modeling a switch as part of its surrounding environment with these methods requires a very large number of unknowns and hence a very large computational time. Moreover, existing codes that assume a typical linear element size of $\lambda / 20$ to $\lambda / 10$ may not necessarily be sufficiently accurate for the MEMS dimensions at hand. Further, since the beam moves while turning on and off, any modeling scheme that meshes the volume within and under the beam, such as FEM or FDTD, requires remeshing of the system for the up and down states. In particular, FDTD is poorly suited to model the deflection of the beam in the down state due to the rectangular grid often used in conjunction with this method.

For the above reasons, the method of moments (MoM) is preferred for the beam portion of the MEMS switch to avoid meshing the region between the beam and the substrate. However, since the switch is part of a complex integrated system such as a multi-layer reconfigurable antenna or part of a frequency-selective surface (FSS), hybrid finite element-boundary integral (FE-BI) methods are preferred to model the complex but stationary MEMS geometry comprised of different materials. Specifically, this paper proposes a combined FE-BI and MoM formulation to be referred to as the extended FE-BI (EFE-BI). This method differs from the conventional hybrid FE-BI formulation [10] since it includes boundary integrals completely external to the FEM domain. This allows for greater flexibility in modeling the deformed 3D surfaces in RF-MEMS switches while it concurrently decreases the computational expense. An efficient preconditioner was also developed to accurately solve the highly ill conditioned matrix system stemming from the extremely small electrical dimensions of RF MEMS [24]. We remark that the theoretical predictions resulting from the proposed integrated full-wave electromagnetic/thermal model are compared with experimental data [25] and found to be in good agreement.

\section{Theory and formulation}

\subsection{Extended FE-BI}

FE-BI is adopted herewith to model the cavity substrate portion of the switch (volume $V_{1}$ enclosed by the surface $S_{1}$ in figure 2),

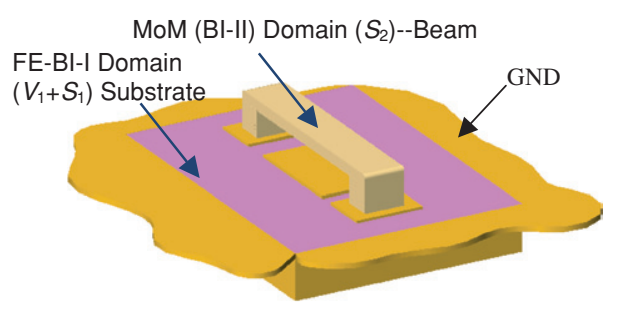

Figure 2. RF-MEMS inline shunt switch model.

whereas MoM is used to treat the MEMS beam (surface $S_{2}$ in figure 2). In the latter case, the resistive sheet model [8] is incorporated to account for the beam's conductivity and thickness. A challenge is to formulate the connectivity of the non-conformal BI surface (on the beam) with the BI bounding the FEM region. Assuming some excitation currents $\left(\vec{J}_{i}, \vec{M}_{i}\right)$, the weak form of the FE-BI [8] for the fixed portion of the switch surface $S_{1}$ (enclosing the volume of $V_{1}$ ) is given by

$$
\begin{aligned}
& \iiint_{V_{1}}\left\{\left(\frac{1}{\mu_{\mathrm{r}}} \nabla \times \vec{E}\right) \cdot(\nabla \times \vec{T})-k_{0}^{2} \varepsilon_{\mathrm{r}} \vec{E} \cdot \vec{T}\right\} \mathrm{d} v \\
& \quad+\mathrm{j} k_{0} Z_{0} \oiint\left(\vec{H}^{S_{1}}+\vec{H}^{S_{2}}\right) \times \hat{n} \cdot \vec{T} \mathrm{~d} s \\
& \quad=-\iiint_{V_{1}}\left\{\mathrm{j} k_{0} Z_{0} \vec{J}^{i}+\nabla \times\left(\frac{1}{\mu_{\mathrm{r}}} \vec{M}^{i}\right)\right\} \cdot \vec{T} \mathrm{~d} v
\end{aligned}
$$

where

$$
\begin{aligned}
& \vec{H}^{S_{1}}=\mathrm{j} \frac{2 k_{0}}{Z_{0}} \iint_{S_{1}} \overrightarrow{\vec{G}}_{0}\left(\vec{r}, \vec{r}^{\prime}\right) \cdot \vec{M}_{S_{1}}\left(\vec{r}^{\prime}\right) \mathrm{d} s^{\prime}, \\
& \vec{H}^{S_{2}}=-\iint_{S_{2}} \nabla \times \overrightarrow{\vec{G}}_{1}\left(\vec{r}, \vec{r}^{\prime}\right) \cdot \vec{J}_{S_{2}}\left(\vec{r}^{\prime}\right) \mathrm{d} s^{\prime} .
\end{aligned}
$$

The various parameters mentioned in the above FE-BI equations are as follows:

- $\vec{M}_{S_{1}}$ : magnetic current density on $S_{1}$ (substrate surface);

- $\vec{J}_{S_{2}}$ : electric current density on $S_{2}$ (MEMS beam);

- $\vec{r}_{i}^{\prime}$ : image point of the source point at $\vec{r}^{\prime}$;

- $k_{0}$ : wavelength number;

- $Z_{0}$ : free-space impedance;

- $\vec{M}(\vec{r})=\vec{E}(\vec{r}) \times \hat{n}$ : equivalent magnetic current density;

- $\vec{J}(\vec{r})=\hat{n} \times \vec{H}(\vec{r})$ : equivalent electric current density;

- $\overrightarrow{\vec{G}}_{0}\left(\vec{r}, \vec{r}^{\prime}\right)=\left(\overrightarrow{\vec{I}}+\frac{1}{k_{0}^{2}} \nabla \nabla\right) G_{0}\left(\vec{r}, \vec{r}^{\prime}\right)$ : free-space dyadic Green function;

- $\overrightarrow{\vec{G}}_{1}\left(\vec{r}, \vec{r}^{\prime}\right)=\left(\overrightarrow{\vec{I}}-\frac{1}{k_{0}^{2}} \nabla \nabla^{\prime}\right)\left(G_{0}\left(\vec{r}, \vec{r}^{\prime}\right)-G_{0}\left(\vec{r}, \vec{r}_{i}^{\prime}\right)\right)$

$+2 \hat{z} \hat{z} G_{0}\left(\vec{r}, \vec{r}_{i}^{\prime}\right):$ half-space dyadic Green function;

- $G_{0}\left(\vec{r}, \vec{r}^{\prime}\right)$ : free-space Green's function; for $k_{0} R \leqslant 0.01$, with its quasi-static approximation obtained by replacing $\mathrm{e}^{-\mathrm{j} k_{0} R}$ with 1 . 
To solve (1) for $\vec{M}_{S_{1}}$ and $\vec{J}_{S_{2}}$, an additional set of equations is required. These equations are generated here by enforcing the boundary conditions on $S_{2}$ (the MEMS beam). Doing so, we get

$$
\begin{gathered}
\hat{n} \times\left[\hat{n} \times\left[\vec{E}^{S_{2}}\left(\vec{J}_{S_{2}}\right)+\vec{E}^{S_{1}}\left(\vec{M}_{S_{1}}\right)\right]\right]=-R \vec{J}_{S_{2}}, \\
\vec{E}^{S_{1}}=-\iint_{S_{1}} \nabla \overrightarrow{\vec{G}}_{0}\left(\vec{r}, \vec{r}^{\prime}\right) \cdot \vec{M}\left(\vec{r}^{\prime}\right) \mathrm{d} s^{\prime}, \\
\vec{E}^{S_{2}}=-\mathrm{j} k_{0} Z_{0} \iint_{S_{2}} \overrightarrow{\vec{G}}_{1}\left(\vec{r}, \vec{r}^{\prime}\right) \cdot \vec{J}_{S_{2}}\left(\vec{r}^{\prime}\right) \mathrm{d} s^{\prime},
\end{gathered}
$$

in which

$$
R=-\frac{\mathrm{i} Z_{0}}{k_{0} d\left(\tilde{\varepsilon}_{\mathrm{r}}-1\right)}, \quad \tilde{\varepsilon}_{\mathrm{r}}=\varepsilon_{\mathrm{r}}-\mathrm{j} \frac{\sigma}{\omega \varepsilon_{0}},
$$

with $d$ and $\sigma$ denoting the beam's thickness and conductivity, respectively.

The substrate is meshed using brick elements to reduce the number of unknowns, with triangular surface elements employed to model the MEMS beam for an accurate representation of the possibly deformed surface. The solution of (1) and (2) then proceeds by expanding the volume and surface tangential electric fields as well as the beam current density using sub-domain basis functions, e.g.,

$$
\begin{aligned}
\vec{E}^{e} & =\sum_{i=1}^{N_{V}} E_{i}^{e} \vec{W}_{i}^{e}, \\
\vec{J}_{S_{2}}^{e} & =\sum_{i=1}^{N_{S_{2}}} J_{i}^{e} \vec{Q}_{i}^{e}, \\
\vec{E}_{S_{1}}^{e} & =\sum_{i=1}^{N_{S_{1}}} E_{i}^{e^{\prime}} \vec{S}_{i}^{e} .
\end{aligned}
$$

Here, $\vec{W}_{i}^{e}, \vec{Q}_{i}^{e}$ and $\vec{S}_{i}^{e}$ represent the basis functions in their respective regions of representation [8]. To enforce current continuity, the surface field basis functions in the FEM domain and the current density basis function (RWG) in the MOM domain [11] are equated at the junction of the BI domain (beam) with the FE-BI domain (substrate). On applying Galerkin's method, the resulting matrix system is of the form

$$
\left[\begin{array}{ccc}
A^{V V} & A^{V S_{1}} & 0 \\
A^{S_{1} V} & A^{S_{1} S_{1}} & A^{S_{1} S_{2}} \\
0 & A^{S_{2} S_{1}} & A^{S_{2} S_{2}}
\end{array}\right]\left\{\begin{array}{c}
E_{n}^{V} \\
E_{n}^{S_{1}} \\
J_{n}^{S_{2}}
\end{array}\right\}=\left\{\begin{array}{c}
b_{m}^{V} \\
b_{m}^{S_{1}} \\
0
\end{array}\right\},
$$

where $\left[A^{V V}\right],\left[A^{V S_{1}}\right],\left[A^{S_{1} V}\right]$ and $\left[A^{S_{1} S_{1}}\right]$ represent the FEBI sub-system for the fixed volume enclosed by $S_{1}$, as shown in figure 2. Typically, $\left[A^{V V}\right],\left[A^{V S_{1}}\right]$ and $\left[A^{S_{1} V}\right]$ are very sparse but $\left[A^{S_{1} S_{1}}\right]$ is dense. Similarly, $\left[A^{S_{1} S_{2}}\right]$ and $\left[A^{S_{2} S_{1}}\right]$ are the sub-matrices representing the interaction between the beam and the BI enclosing the substrate. They are dense and so is $\left[A^{S_{2} S_{2}}\right]$, a sub-matrix representing the discrete MoM system. The elements for each sub-matrix are given by

$$
\begin{gathered}
\left(A_{i j}^{e}\right)^{V}=\iiint_{V_{e}}\left\{\left(\frac{1}{\mu_{\mathrm{r}}}\left(\nabla \times \vec{W}_{i}^{e}\right) \cdot\left(\nabla \times \vec{W}_{j}^{e}\right)\right)\right. \\
\left.-k_{0}^{2} \varepsilon_{\mathrm{r}} \vec{W}_{i}^{e} \cdot \vec{W}_{j}^{e}\right\} \mathrm{d} v,
\end{gathered}
$$

$$
\begin{aligned}
& \left(A_{i j}^{e}\right)^{S_{1} S_{1}}=2 k_{0}^{2} \iint_{S_{1}^{e}}\left[\hat{n} \times \vec{S}_{j}^{e}(\vec{r})\right] \\
& \cdot\left\{\iint_{S_{1}^{e^{\prime}}} \overrightarrow{\vec{G}}\left(\vec{r}, \vec{r}^{\prime}\right) \cdot\left[\vec{S}_{j}^{e^{\prime}}\left(\vec{r}^{\prime}\right) \times \hat{n}\right] \mathrm{d} s^{\prime}\right\} \mathrm{d} s \\
& \left(A_{i j}^{e}\right)^{S_{1} S_{2}}=\mathrm{j} k_{0} Z_{0} \iint_{S_{1}^{e}}\left[\hat{n} \times \vec{S}_{j}^{e}(\vec{r})\right] \\
& \quad \times \iint_{S_{2}^{e^{\prime}}}\left\{\left[\nabla G_{0}\left(\vec{r}, \vec{r}^{\prime}\right)-\nabla G_{0}\left(\vec{r}, \vec{r}_{i}^{\prime}\right)\right]\right. \\
& \left.\quad \times \vec{Q}_{i}^{e^{\prime}}\left(\vec{r}^{\prime}\right)+2 Q_{i z}^{e^{\prime}}\left(\vec{r}^{\prime}\right) \cdot \nabla G_{0}\left(\vec{r}, \vec{r}_{i}^{\prime}\right) \times \hat{z}\right\} \mathrm{d} s^{\prime} \mathrm{d} s
\end{aligned}
$$

$$
\begin{aligned}
& \left(A_{i j}^{e}\right)^{S_{2} S_{1}}=-2 \iint_{S_{2}^{e}}\left[\vec{Q}_{j}^{e}(\vec{r})\right] \\
& \quad \cdot\left\{\iint_{S_{1}^{e^{\prime}}} \nabla G_{0}\left(\vec{r}, \vec{r}^{\prime}\right) \times\left(\vec{S}_{i}^{e^{\prime}}\left(\vec{r}^{\prime}\right) \times \hat{n}^{\prime}\right) \mathrm{d} s^{\prime}\right\} \mathrm{d} s \\
& \left(A_{i j}^{e}\right)^{S_{2} S_{2}}=-\mathrm{j} k_{0} Z_{0} \iint_{S_{2}^{e}}\left[\vec{Q}_{j}^{e}(\vec{r})\right] \\
& +\left\{\iint_{S_{2}^{e^{\prime}}}\left[G_{0}\left(\vec{r}, \vec{r}^{\prime}\right)-G_{0}\left(\vec{r}, \vec{r}_{i}^{\prime}\right)\right] \cdot \vec{Q}_{i}^{e^{\prime}}\left(\vec{r}^{\prime}\right) \mathrm{d} s^{\prime}\right. \\
& +\iint_{S_{2}^{e^{\prime}}}\left[\frac{\nabla \nabla}{k_{0}^{2}} G_{0}\left(\vec{r}, \vec{r}^{\prime}\right)+\frac{\nabla \nabla^{\prime}}{k_{0}^{2}} G_{0}\left(\vec{r}, \vec{r}_{i}^{\prime}\right)\right] \cdot \vec{Q}_{i}^{e^{\prime}}\left(\vec{r}^{\prime}\right) \mathrm{d} s^{\prime} \\
& \left.+2 \iint_{S_{2}^{e^{\prime}}} \hat{z} \cdot G_{0}\left(\vec{r}, \vec{r}_{i}^{\prime}\right) \cdot Q_{i z}^{e^{\prime}}\left(\vec{r}^{\prime}\right) \mathrm{d} s^{\prime}\right\} \mathrm{d} s \\
& +R \iint_{S_{2}^{e^{\prime}}} \vec{Q}_{j}^{e}(\vec{r}) \vec{Q}_{i}^{e}(\vec{r}) \mathrm{d} s
\end{aligned}
$$

$\left(b_{m}^{V}\right)^{e}=-\iiint_{V_{e}} \vec{W}_{j}^{e} \cdot\left\{j k_{0} Z_{0} \vec{J}^{i}+\nabla \times\left(\frac{1}{\mu_{\mathrm{r}}} \vec{M}^{i}\right)\right\} \mathrm{d} v$,

$\left(b_{m}^{S_{1}}\right)^{e}=0$.

\subsection{Thermal modeling}

Once the current densities are obtained via the EFE-BI analysis, heating in the beam can be estimated using the steadystate heat equation

$$
-\nabla(\kappa \nabla T)=J^{2} \rho,
$$

where $\kappa$ is the thermal conductivity, $T$ is the temperature, $J$ is the RMS current density through the beam and $\rho$ is the electrical resistivity. The steady-state heat equation is adopted since the signal/current frequency is much higher than that of the thermal response (typically on the order of $\mathrm{kHz}$ ).

To obtain the temperature rise in the beam, we adapted a two-dimensional FEM solution of (6) [7] to calculate the temperature distribution in the $x-y$ plane, as shown in figure 3 . In our model, we assumed a constant temperature across the beam thickness. Also, we ignored effects due to radiative and convective heat transfer (since they are rather insignificant). However, our model did include the conduction of heat through the thin air gap under the beam. In the following, we discuss how the heat model is integrated with the EFE-BI for a complete EM-heat analysis of MEMS switches. 


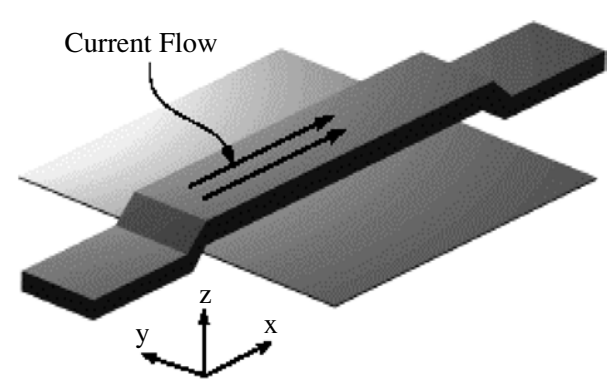

Figure 3. Current distribution along the beam.

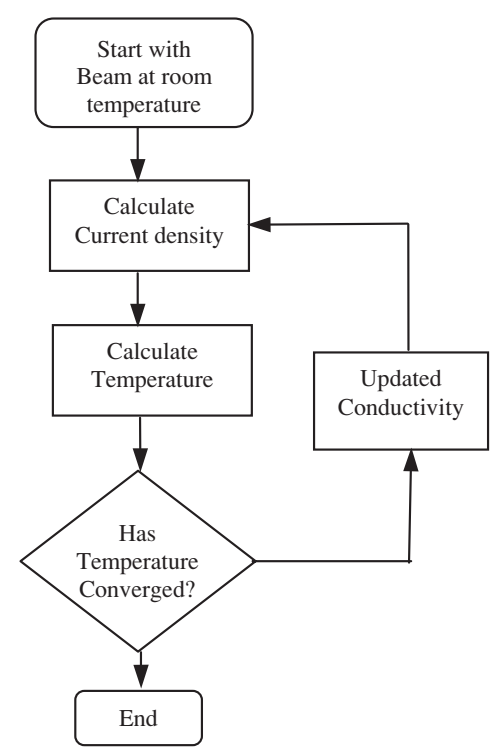

Figure 4. Flowchart for calculating switch temperature rise.

\subsection{Integration of thermal and EM models}

As is well known, heat dissipation that dictates temperature rise scales with the electrical resistivity of the beam, a function of temperature. An iterative procedure (see figure 4) was employed to reach temperature convergence. While this procedure is quite simple, we also found that one to three iterations are sufficient (in most cases) to reach convergence to within $1 \%$.

\section{Integral accuracy}

Because RF-MEMS switches are electrically very small at radio frequency (RF) bands (typically, $L$ to $X$ bands), the accuracy of the matrix elements in (4) must be carefully computed, particularly for those in the boundary integral submatrices. This is a unique issue for MEMS modeling and must be addressed for a reliable analysis. In this paper, we combined both quasi-static as well as known full-wave integral evaluation to generate accurate matrix entries (figure 5).

For the matrix elements in $A^{S_{1} S_{1}}$, analytical expressions are given in [8]. Also the elements of the coupling matrices $A^{S_{1} S_{2}}$ and $A^{S_{2} S_{1}}$ can be readily integrated numerically since they have no singularities. However, accuracy becomes a serious issue for the self-cell entries and the quasi-static approximated non-self-cell elements in $A^{S_{2} S_{2}}$. Unless the accuracy of these elements is carefully controlled, the highly ill conditioned

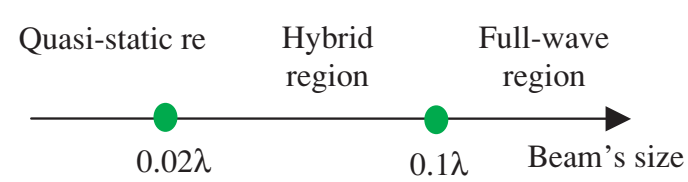

Figure 5. Employed approximation versus beam dimension.

Table 1. Self-cell integral formulation modification [14].

\begin{tabular}{llll}
\hline & \multicolumn{2}{c}{$I_{\text {iip }}$} \\
\cline { 2 - 4 } Node no & \multicolumn{2}{c}{ Analytical results [14] } & $\begin{array}{l}\text { Semi-analytical results [13] } \\
\text { (number of integration points) }\end{array}$ \\
\hline$i=1$ & Before & 14.9012854 & $14.410755(5)$ \\
& After & 14.3389690 & $14.346337(9)$ \\
& & & $14.339979(15)$ \\
$i=2$ & Before & 14.4442568 & $14.381827(5)$ \\
& After & 14.3100099 & $14.317372(9)$ \\
& & & $14.311019(15)$ \\
$i=3$ & Before & 11.8907294 & $12.588863(5)$ \\
& After & 12.5872927 & $12.587479(9)$ \\
& & & $12.587321(15)$ \\
\hline
\end{tabular}

matrix leads to large inaccuracies within the solution of the current densities or fields [24]. In this work, the non-selfcell integrals are evaluated using a semi-analytical approach [13] to increase speed and accuracy. For the same reason, the self-cell integrals are evaluated analytically following [14]. However, prior to using the expressions in [14], two misprints were corrected. In (26) of [14], the two terms without a logarithm in the numerator should carry a coefficient of 60 rather than 120 in their denominators. Namely, the integration of $I_{i i p}=\int_{A} \int_{A} N_{i}^{\prime} N_{i} \frac{1}{\left|\vec{r}-\vec{r}^{\prime}\right|} \mathrm{d} a \mathrm{~d} a^{\prime}$, where $N_{i}^{\prime}$ and $N_{i}(i=1,2$, 3 ) are the simplex coordinates on the source and observation triangles, must be corrected to read

$$
\begin{aligned}
\frac{1}{4 A^{2}} & \int_{A} \int_{A} N_{i}^{\prime} N_{i} \frac{1}{\left|\vec{r}-\vec{r}^{\prime}\right|} \mathrm{d} a \mathrm{~d} a^{\prime} \\
& =\frac{\ln \left(\frac{b+\sqrt{a c}}{b-c+\sqrt{c} \sqrt{a-2 b+c}}\right)}{40 \sqrt{c}}+\frac{\ln \left(\frac{-b+c+\sqrt{c} \sqrt{a-2 b+c}}{-b+\sqrt{a c}}\right)}{40 \sqrt{c}} \\
& +\frac{\sqrt{a} \sqrt{a-2 b+c}-\sqrt{c} \sqrt{a-2 b+c}}{60(a-2 b+c)^{1.5}}+\frac{(2 a-5 b+3 c)}{120(a-2 b+c)^{1.5}} \\
& \times \ln \left(\frac{(a-b+\sqrt{a} \sqrt{a-2 b+c})(c-b+\sqrt{c} \sqrt{a-2 b+c})}{(b-a+\sqrt{a} \sqrt{a-2 b+c})(b-c+\sqrt{c} \sqrt{a-2 b+c})}\right) \\
& +\frac{-\sqrt{a} \sqrt{c}+\sqrt{a} \sqrt{a-2 b+c}}{60(a)^{1.5}}+\frac{(2 a+b)}{120(a)^{1.5}} \\
& \times \ln \left(\frac{(a-b+\sqrt{a} \sqrt{a-2 b+c})(b+\sqrt{c} \sqrt{a})}{(b-a+\sqrt{a} \sqrt{a-2 b+c})(-b+\sqrt{c} \sqrt{a})}\right)
\end{aligned}
$$

Table 1 shows a comparison among the analytical form in [14] (before and after correcting the misprints) and the semianalytical formulation [13]. These results clearly show that our new expression (7) is indeed the correct one. We note that the integration triangle used here has nodes at $P_{1}(62.5,25,0)$, $P_{2}(62.5,25,2)$ and $P_{3}(62.5,37.5,0)$.

The data reported in figure 6 show that the integration of $\int_{A} \int_{A} \frac{1}{\left|\vec{r}-\vec{r}^{\prime}\right|} \mathrm{d} a \mathrm{~d} a^{\prime}$ for disjoint triangles based on the semianalytical formulae in [13] gives much higher accuracy by 


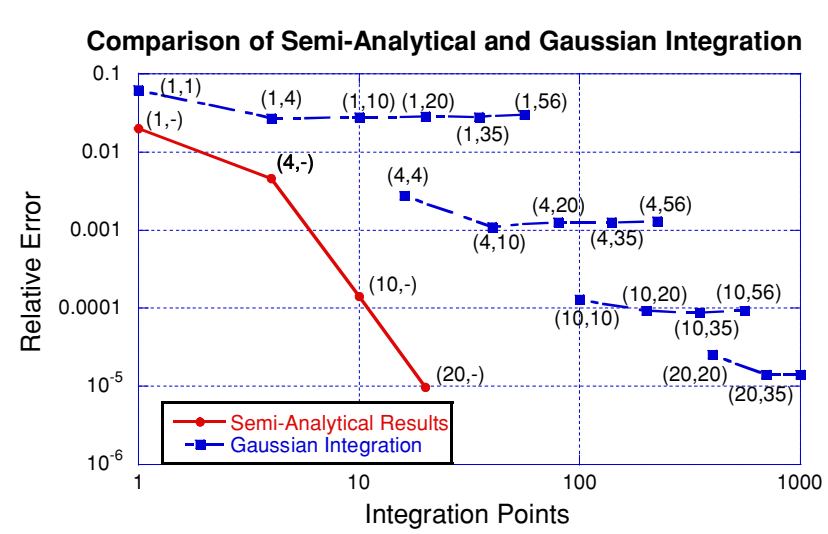

Figure 6. Maximum relative errors of the semi-analytical [13] and Gaussian [15] integrations for disjoint triangles.

Table 2. Comparison cases.

\begin{tabular}{lcll}
\hline Case & $\begin{array}{l}\text { Frequency } \\
(\mathrm{GHz})\end{array}$ & Beam size $(\mu \mathrm{m})$ & $\begin{array}{l}\text { Compared } \\
\text { with }\end{array}$ \\
\hline $\mathrm{a}$ & 3 & $10000 \times 5000(\lambda / 10 \times \lambda / 20)$ & AMFIA \\
$\mathrm{b}$ & 300 & $75 \times 50(3 \lambda / 40 \times \lambda / 20)$ & HFSS \\
$\mathrm{c}$ & 3000 & $75 \times 50(3 \lambda / 4 \times \lambda / 2)$ & HFSS \\
$\mathrm{d}$ & 20 & $75 \times 50(\lambda / 200 \times \lambda / 300)$ & Static $(\mathrm{dc})$ \\
\hline
\end{tabular}

using fewer integration points as compared to direct Gaussian integration [15]. In addition, the semi-analytical integral forms take much less time to evaluate since one of the integrals is given in analytical form [13]. We remark that the relative error given in figure 6 was referenced to an adaptive integration approach.

\section{Validation examples}

\subsection{Validation using commercial codes}

A computer code using the preceding EFE-BI formulation was developed to determine the current distribution on the RF-MEMS switch structures. At different frequencies, the results are compared with the code AMFIA developed in [12] as well as the commercial software HFSS v9.2 and a static (dc) approximation.

The comparisons are made for the four cases shown in table 2. Due to difficulties in the convergence of the conventional FE-BI and FEM for electrically small MEMS structures, the frequency and beam size are selected such that their electrical length is large (approximately between $\lambda / 50$ and $\lambda$ ), resulting in a rather unrealistically large beam size for case (a) as depicted in table 2. The same is true for cases (b) and (c) in table 2. This is done because in realistic beam sizes and frequencies, the electrical length is on the order of $\lambda / 100$, i.e. too small for conventional methods to converge and lead to unreliable answers. Thus, to qualitatively assess the accuracy of our method for such very small electrical lengths, a static (dc) approximation was used. This evaluation refers to case (d) in table 2 and employs realistic dimensions at typical RF frequencies. We remark that the junction between the beam and the substrate is ignored in case (a) due to the limitation of the FE-BI formulation used in the AMFIA software. That is, the 'beam' is an 'RF strip' floating above the substrate with no electrical connection to the substrate.

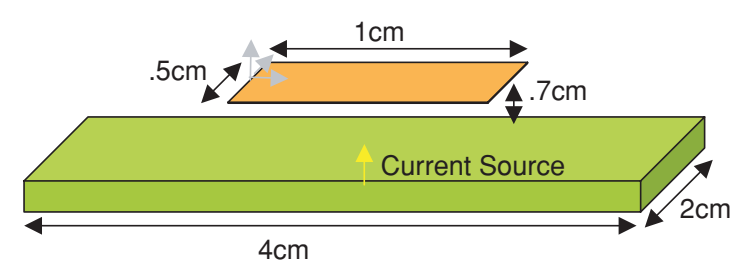

Figure 7. RF strip floating on a substrate for comparison with case (a) in table 2 .

Figure 7 shows the dimensions of the 'RF strip' along with the substrate for case (a) in table 2. The excitation is a vertical (to the beam) current dipole operating at $3 \mathrm{GHz}$ and placed at the center of the substrate. Figure 8 shows the current distribution along the strip length (values at the middle of the strip) using the full-wave formulation based on our EFE-BI code versus the conventional FE-BI (AMFIA). The comparisons demonstrate good agreement between the two methods. As compared to the conventional FE-BI, we remark that EFE-BI uses much fewer unknowns and less computation time, since the gap between the beam and the substrate is not meshed.

Figure 9 shows an RF-MEMS switch for the comparison of the cases (b)-(d) depicted in table 2. As shown, the excitation is represented by a five-probe feed (impressed electrical current) [8]. For our EFE-BI analysis, a quasi-static approximation and a half-triangle basis function were used to model the junction between the two domains. Figure 10 shows a comparison of the current density distribution along the beam length for cases (b) and (c). Again, good agreement between the two methods is obtained. Moreover, as in case (a), the convergence of our EFE-BI analysis is significantly faster than that of the conventional FE-BI technique. In case (b), for instance, HFSS required 122834 tetrahedral elements and $17.5 \mathrm{~h}$ to converge, whereas EFE-BI used only 821 unknowns and $24 \mathrm{~min}$ to solve. While our EFE-BI converges quickly even for much lower frequencies, such a comparison at low frequencies was not made since HFSS could not converge.

Figure 11 shows a comparison of the current distribution along the width for case (d). The results again agree quite well, even though minor edge effects are observed at both sides of the beam in the EFE-BI solution. This indicates that at frequencies lower than $20 \mathrm{GHz}$, the static approximation is a reasonable way to evaluate the current distribution for RF-MEMS switches below $100 \mu \mathrm{m}$ in size. For the 20 $100 \mathrm{GHz}$ frequency range, the static approximation is no longer accurate due to significant edge effects. In this range, the conventional numerical codes such as ANSYS that employs quasi-static approximations could also not converge since the beam's dimensions are outside their validity range. Hence, the EFE-BI solution with combined quasi-static and full-wave integral evaluation (figure 5) is a necessary approach.

\subsection{Experimental validation}

Recently, we also performed experimental studies on the selfheating of surface-micromachined gold RF-MEMS structures suspended on a substrate, shown in figure 12. The 

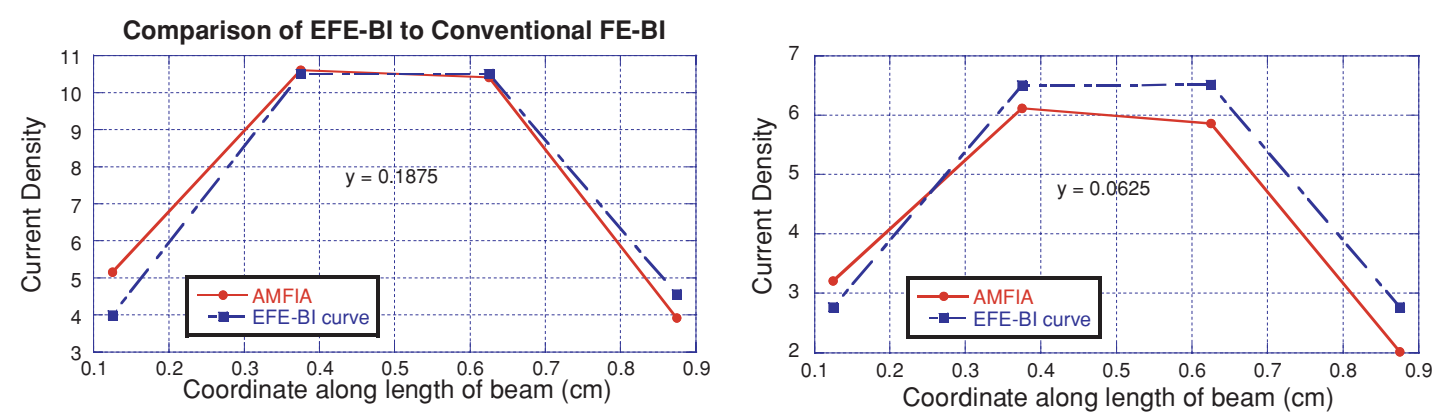

Figure 8. Current distribution at the center along the length of the RF strip in figure 7 (gap $=0.7 \mathrm{~cm}$ ).

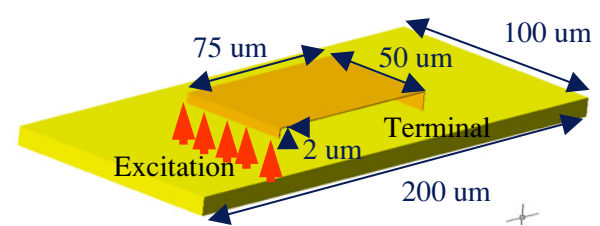

Figure 9. RF-MEMS switch for comparison among cases (b)-(d) in table 2 .

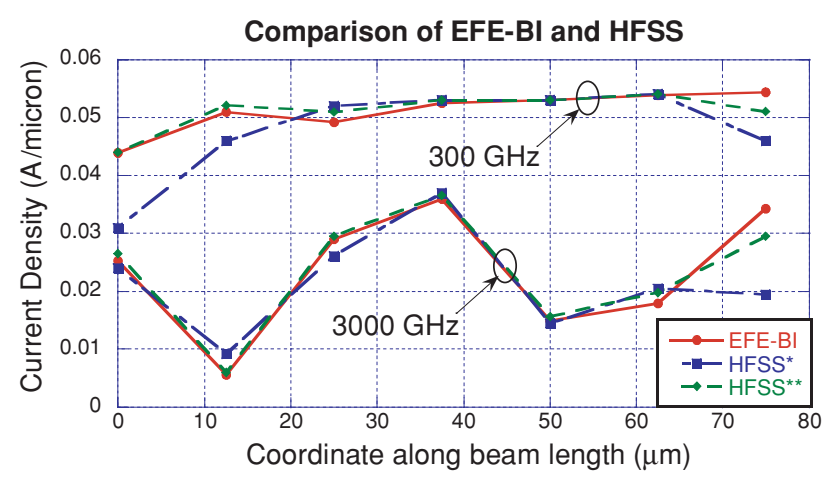

Figure 10. Current distribution along the length of the RF-MEMS switch in figure 8 . $\left({ }^{*}\right)$ Simulation for a bounding box two times larger than the geometry; $\left(^{* *}\right)$ bounding box five times larger than the geometry.

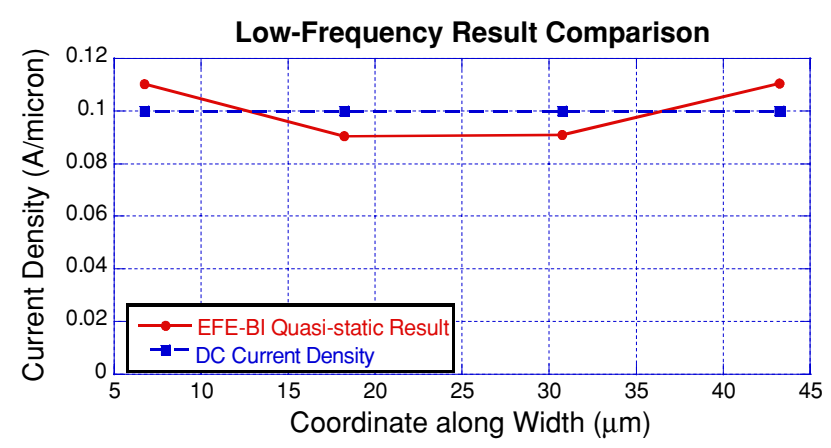

Figure 11. Current distribution along the width of the beam.

experimental data refer to a device subjected to an RF signal and can be used to validate our model predictions for the selfheating of an RF-MEMS switch in its 'up' state. The details of the design, fabrication and RF/thermal characterization of the testing device can be found in [25]. In brief, we measured the spatially averaged temperature increase in a MEMS RF structure at various frequencies and RF input power using

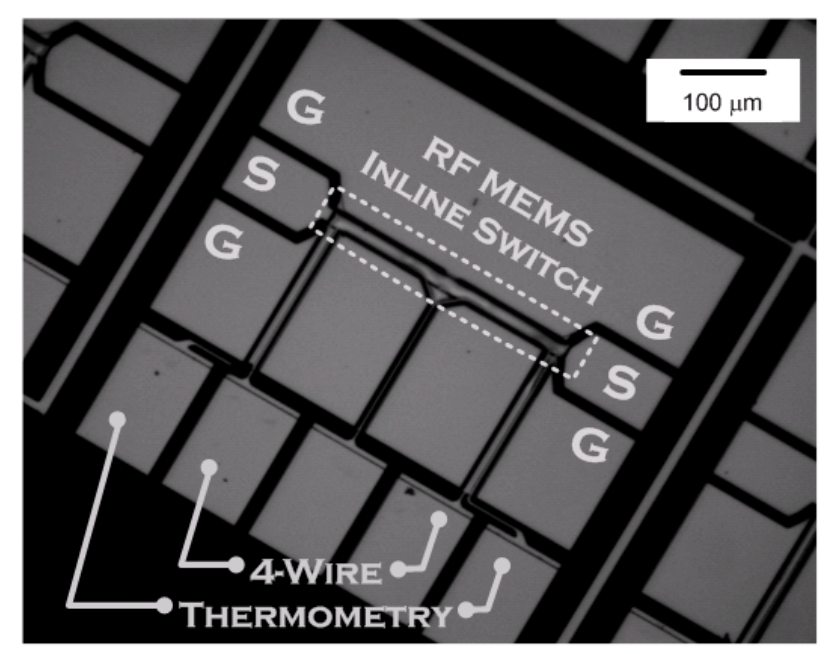

Figure 12. SEM image of a surface-micromachined RF-MEMS inline switch.

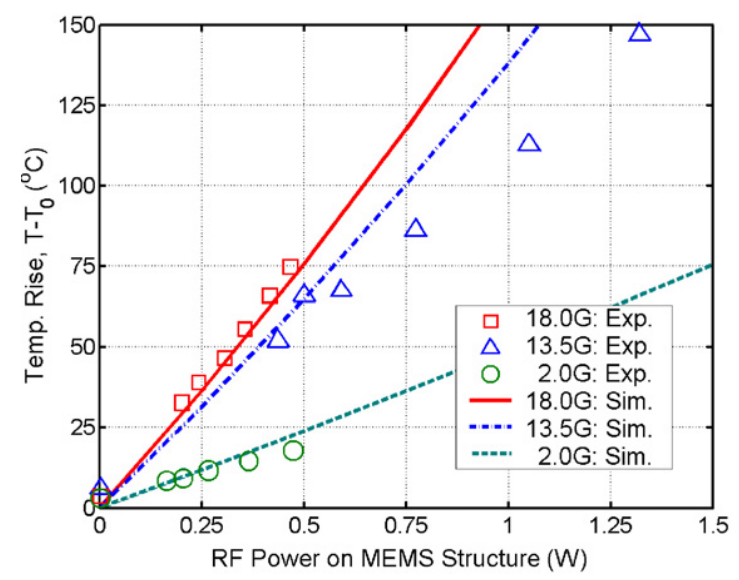

Figure 13. Model predictions and experimental data of the spatially averaged temperature rise in a test device simulating the up-state of an RF-MEMS switch. Comparisons are shown as a function of input power at three different frequencies $(2 \mathrm{GHz}, 13.5 \mathrm{GHz}$ and $18 \mathrm{GHz})$. The experimental data are from [25].

a dc electrical resistance thermometry technique, specially developed for an RF setup. Figure 13 compares our model predictions and experimental data, clearly demonstrating that our numerical predictions are in good agreement to the experimental data. 

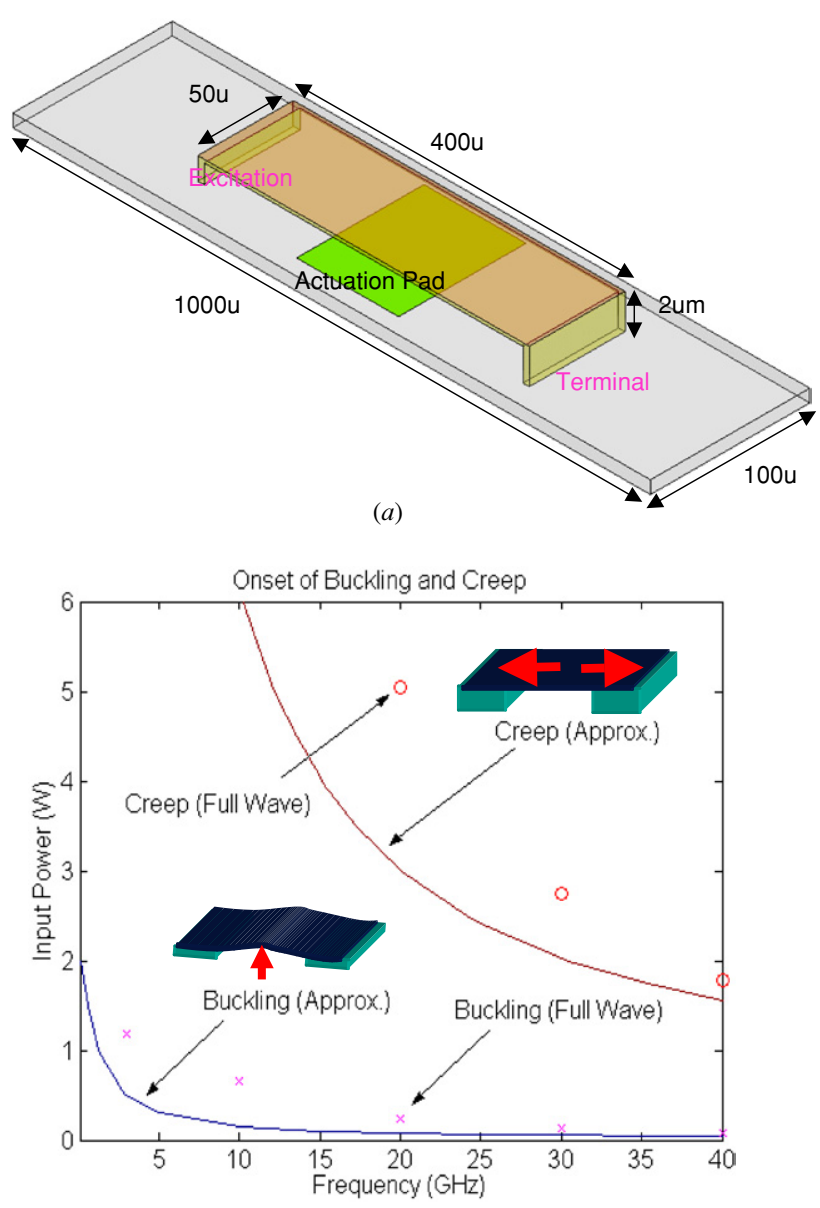

(b)

Figure 14. (a) Switch model and (b) predicted onset of buckling and creep.

\section{Failure mechanisms for RF-MEMS switches}

The integrated electrothermal model using the EFE-BI method presented in section 2 will now be used to predict the failure of an RF-MEMS switch due to creep and bucking at elevated device temperatures. The results are compared with those in our previous work [16] which employed an approximate electromagnetic model.

Creep is a time-dependent plastic deformation of the metal under stresses well below their yield strength. It is observed when the beam's metal is subjected to temperatures above one-half of its melting point [17]. Hence, a gold switch with a melting point of $1340 \mathrm{~K}$ is likely to fail over time if the temperature exceeds $670 \mathrm{~K}$, or $392{ }^{\circ} \mathrm{C}$.

On the other hand, buckling occurs when compressive stresses in the beam introduce instability while deflecting, causing erratic and unpredictable switch behavior. Buckling due to thermal stresses is predicted via the Euler buckling formula [7]

$$
\varepsilon_{\mathrm{cr}}=\frac{C \pi^{2} t^{2}}{12 l^{2}},
$$

where $\varepsilon_{\mathrm{cr}}$ is the critical buckling strain in the beam, $t$ is the beam thickness, $l$ is the beam length and $C$ is 4 for a fixedfixed beam. Hence, for a beam with $l=400 \mu \mathrm{m}$ and $t=2 \mu \mathrm{m}$, the critical buckling strain is $8.22 \times 10^{-5}$. Buckling will occur when $\varepsilon_{\text {th }}=\varepsilon_{\mathrm{cr}}$, where the thermal strain $\varepsilon_{\mathrm{th}}$ in the beam is given by

$$
\varepsilon_{\mathrm{th}}=\frac{1}{l} \int_{0}^{l} \alpha \Delta T \mathrm{~d} x
$$

where $\alpha$ is the thermal expansion coefficient $\left(13.8 \times 10^{-6}{ }^{\circ} \mathrm{C}^{-1}\right.$ for gold) and $\Delta T$ is the change in temperature.

Figure 14(a) displays a MEMS switch with a glass $\left(\varepsilon_{\mathrm{r}}=4\right)$ substrate $1000 \times 100 \times 2 \mu \mathrm{m}^{3}$ and a gold beam $400 \times 50 \times 2 \mu \mathrm{m}^{3}$. Figure $12(b)$ gives the corresponding comparisons of the two failure mechanisms using full-wave (with EFE-BI) and approximate modeling [16]. For this geometry, buckling is the dominant failure mechanism at all frequencies and power levels. It is observed that the EFEBI full-wave model consistently predicts lower temperatures than the approximate model [21], and this is likely due to the current approximations near the edges of the beam used in the approximate model. Nevertheless, the trend remains the same: failure is more likely as either power or frequency increases (power having the stronger influence). That is, at typical RF frequencies ( $\mathrm{X}$ band and beyond), failure due to buckling can occur with very little power as compared to the case when the same switch is operated near dc. In fact, the buckling and yielding failure phenomena were observed during and after the RF self-heating, and refer to the device operating below $19 \mathrm{GHz}$ and with an RF power near $0.5 \mathrm{~W}$ [25]. Such failure phenomena could be suppressed by designing a switch having wider, shorter and thicker beam and by controlling device processing condition to introduce tensile residual stress within the beam.

\section{Conclusion}

We developed an extended FE-BI (EFE-BI) formulation for the calculation of the current density distribution on the beam of inline shunt RF-MEMS switches. A key aspect of the EFE-BI is the use of accurate matrix element approximations which combined semi-analytical integral formulae to address the small dimensions associated with RF-MEMS switches, especially at UHF to X-band frequencies. This allowed for practical RF-MEMS modeling where the other methods failed due to highly ill conditioned matrix systems [24] caused by the small dimensions. Validation examples showed that the accuracy and efficiency of our method compared quite well to the conventional FE-BI, commercial HFSS, static approximations (when appropriate) and measurements. Also, the EFE-BI method generated accurate results much faster and at lower frequencies than previous methodologies. Because the air around the MEMS switch is not meshed, significant savings in computer time and memory were observed. Integration of this rigorous full-wave EM mythology with 2D FEM thermal analysis was used to predict the failure mechanisms for a practical MEMS switch and obtain good matching result. We remark that although the focus was on an inline and up-state shunt switch, the method is equally applicable to other type of switches, possibly coupled with future design optimization tools aimed at maximizing 
the power-handling capability and reliability of RF-MEMS switches.

\section{References}

[1] Yao J J 2000 RF-MEMS from device perspective J. Micromech. Microeng. 10 R8-38

[2] Pacheco S, Nguyen C T and Katehi L P B 1998 Micromechanical electrostatic K-band switches 1998 IEEE MTT-S Microwave Symp. Digest 3 1569-72

[3] Zavracky P M, McGruer N E, Morrison R H and Potter D 1999 Microswitches and microrelays with a view toward microwave applications Int. J. RF Microw. Theory Tech. 9 338-47

[4] Rebeiz G M and Muldavin J B 2001 RF MEMS switches and switch circuits IEEE Microw. Mag. 2 59-71

[5] Duffy S, Bozler C, Rabe S, Knecht J, Travis L, Wyatt P, Keast C and Gouker M 2001 MEMS microswitches for reconfigurable microwave circuitry IEEE Microw. Wirel. Compon. Lett. 11 106-8

[6] Muldavin J B and Rebeiz G M 2001 Inline capacitive and dc-contact MEMS shunt switches IEEE Microw. Wirel. Compon. Lett. $11334-6$

[7] Jensen B D, Saitou K, Volakis J L and Kurabayashi K 2003 Impact of skin effect on thermal behavior of RF MEMS switches 6th ASME-JSME Thermal Engineering Joint Conference (Paper no TED-AJ03-420)

[8] Volakis J L, Chatterjee A and Kempel L C 1998 Finite Element Method of Electromagnetics (New York: IEEE)

[9] Taflove A and Hagness S C 2000 Computational Electrodynamics: The Finite-Difference Time-Domain Method (Boston, MA: Artech House Publishers)

[10] Jin J M, Volakis J L and Collins J D 1991 A finite element-boundary integral method for scattering and radiation by two- and three-dimensional structures IEEE Antennas Propag. Mag. 33 22-32

[11] Lee J-F, Lee R and Burkholder R J 2003 Loop star basis functions and a robust preconditioned for EFIE scattering problems IEEE Trans. Antennas Propag. 51 1855-63

[12] Kindt R, Sertel K, Topsakal E and Volakis J L 2003 Array decomposition method for the accurate analysis of finite arrays IEEE Trans. Antennas Propag. 51 1364-72

[13] Wang Z, Volakis J, Saitou K and Kurabayashi K 2003 Comparison of semi-analytical formulations and Gaussian quadrature rules for quasi-static double surface potential integrals IEEE Antenna Propag. Mag. 45 96-102
[14] Eibert T F and Hansen V 1995 On the calculation of potential integrals for linear source distributions on triangle domains IEEE Trans. Antennas Propag. 42 1499-502

[15] Ariconi P, Bressan M and Perregrini L 1997 On the evaluation of the double surface integrals arising in the application of the boundary integral method to 3-D problems IEEE Trans. Microw. Theory Tech. 45 436-9

[16] Jensen B D, Saitou K, Volakis J L and Kurabayashi K 2003 Fully integrated electrothermal multi-domain modeling of RF MEMS switches IEEE Microw. Wirel. Compon. Lett. 13 364-6

[17] Barret C R, Nix W D and Tetelman A S 1973 The Principles of Engineering Materials (Englewood Cliffs, NJ: Prentice-Hall)

[18] Wang Z, Jensen B, Volakis J, Saitou K and Kurabayashi K 2003 Analysis of RF-MEMS switches using finite element-boundary integration with moment method Proc. IEEE Society International Conference on Antennas and Propagation (22-27 June) vol 2 pp 173-6

[19] Rao S M, Wilton R and Glisson A W 1982 Electromagnetic scattering by surfaces of arbitrary shape IEEE Trans. Antennas Propag. 30 409-18

[20] Rao S M, Glisson A W, Wilton D R and Vidula B S 1979 A simple numerical solution procedure for static problems involving arbitrary-shaped surfaces IEEE Trans. Antennas Propag. 27 604-8

[21] Jensen B D, Wang Z, Chow L, Saitou K, Kurabayashi K and Volakis J L 2003 Integrated electrothermal modeling of RF MEMS switches for improved power handling capability 2003 IEEE Topical Conference on Wireless Communication Technology (Hawaii, 15-17 October)

[22] Jensen B D, Wang Z, Saitou K, Volakis J and Kurabayashi K 2003 Simultaneous electrical and thermal modeling of a contact-type RF MEMS switch 2003 ASME International Mechanical Engineering Congress and $R \& D$ Expo (Washington, DC, 15-21 November)

[23] Graglia R D 1993 On the numerical integration of linear shape functions times the 3-D Green's function or its gradient on a pane triangle IEEE Trans. Antennas Propag. 41 1448-55

[24] Wang Z, Jensen B, Volakis J, Saitou K and Kurabayashi K 2004 Highly-coupled preconditioner for EFE BI arising in RF MEMS switch analysis Proc. IEEE AP-S International Symposium and USNC/CNC/URSI National Radio Science Meeting (Monterey, CA, 20-26 June)

[25] Chow L L W, Wang Z, Jensen B D, Saitou K, Volakis J L and Kurabayashi K Skin-effect self-heating in air-suspended RF MEMS transmission-line structures J. Microelectromech. Syst. (submitted) 